\title{
West Lake Staging: A New Staging System Orchestrated by X-Ray and MRI on Knee Osteoarthritis
}

\section{Jiaxin Huang}

Zhejiang Chinese Medical University

\section{Xi Chen}

Zhejiang University

\section{Mengting Xia}

Zhejiang Chinese Medical University

\section{Shuaijie Lv}

The First Affiliated Hospital of Zhejiang Chinese Medical University

Peijian Tong ( $\sim$ tongpeijian@163.com )

the First Affiliated hospital of Zhejiang Chinese Medical University https://orcid.org/0000-0003-09488674

\section{Research article}

Keywords: osteoarthritis, knee, X-ray, MRI, Kellgren - Lawrence staging

Posted Date: December 29th, 2020

DOl: https://doi.org/10.21203/rs.3.rs-135790/v1

License: (c) (i) This work is licensed under a Creative Commons Attribution 4.0 International License. Read Full License

Version of Record: A version of this preprint was published at Journal of Orthopaedic Surgery on September 1st, 2021. See the published version at https://doi.org/10.1177/23094990211049587. 


\section{Abstract}

Background: Although widely used of Kellgren - Lawrence (KL) staging system on knee osteoarthritis (KOA), it still had some disadvantages. The purpose of this study was to investigate the differences on Xray and MRI among each stage of KOA and further propose a new staging system called West Lake (WL) staging.

Methods: A cross-sectional study was conducted on patients with KOA. KL stages were defined by two qualified orthopedic surgeons. Joint space widths (JSW) were measured on X-rays, whereas cartilage injuries $(\mathrm{Cl})$ and bone marrow lesions $(\mathrm{BML})$ were evaluated on MRI. The differences of them across the groups were calculated by T-test. Receiver operating characteristic (ROC) curves were rendered to obtain the areas under the curves (AUC), Youden index and corresponding cut-off points. Agreement Index (KAPPA) was used to analyze the consistency of KL stages and WL stages assessed by two qualified orthopedic surgeons.

Results: Eventually, a total of 220 patients met the criteria. There were significant differences on JSW, Cl and $\mathrm{BML}$ between $\mathrm{KL} \mathrm{II} / \mathrm{III}$ as well as $\mathrm{KL}$ III/IV. In $\mathrm{KL} \mathrm{II} / \mathrm{III}$, the AUC of JSW, Cl, BML was $0.99,0.76,0.71$ and the Youden index was $0.94,0.38,0.45$, meanwhile the cut-off points were $£ 5.1 \mathrm{~mm},>1,>2$. In $\mathrm{KL} \mathrm{III} / \mathrm{IV}$, the AUC of JSW, $\mathrm{Cl}, \mathrm{BML}$ was $0.96,0.79,0.74$ and the Youden index was $0.84,0.58,0.38$, meanwhile the cutoff points were $£ 3.2 \mathrm{~mm},>3,>4$. The Kappa coefficients of KL stages and WL stages were 0.31 and 0.76 .

Conclusion: The WL staging was described as follows: Grade 0, normal X-ray. Grade 1, X-ray shows osteophyte but no joint space narrow, normal MRI or MRI shows cartilage degeneration and only 1 or 2 sections are involved in BML. Grade 2, X-ray shows joint space narrow, MRI shows cartilage defect but no full-thickness cartilage defect, meanwhile 3 or 4 sections are involved in BML. Grade 3, X-ray shows serious joint space narrow even JSW disappeared, MRI shows full-thickness cartilage defect, more than 4 sections are involved in BML.

Trial registration: The study was approved by the First Affiliated Hospital of Zhejiang Chinese Medical University (2018-ZX-026-01).

\section{Background}

Knee osteoarthritis (KOA) is a common chronic disease manifested by articular cartilage degradation, bone remodeling, osteophyte formation, and eventually the loss of joint space widths (JSW) [1]. Indisputably, KOA affected the function of joints and reduce the quality of life, even led to disability, finally incurred enormous social and economic burdens [2-4].

Although the long and wide implementation since its generation in 1957, Kellgren - Lawrence (KL) staging system of the knee still had some deficiencies which could affect the surgeons' judgment of the severity of KOA and the choice of therapeutic schedule [5]. KL staging based on radiographs has gradually exerted the shortcoming in terms of the ambiguous differentiation due to its non-quantitative depiction 
on the morphological change especially in patients at the early stage of KOA, leading to the uncertainty in hierarchical remedy. In recent decades, despite numerous attempts based on X-rays on modifying this classification, none has been widely recognized, moreover, the non-quantitative traits of KL staging were hardly radically revolutionized $[6,7]$.

With the popularity of MRI, the perception of KOA has been rapidly upgraded, pathological change including cartilage injuries $(\mathrm{Cl})$, bone marrow lesions $(\mathrm{BML})$, etc. were realized their diagnostic significance [8]. However, an integrated algorithm encompassing X-rays and MRI considering JSW, $\mathrm{Cl}$, BML has never been generated for better KOA grading.

Therefore, in this study, we aimed at investigating the differences on X-ray and MRI among each stage of $\mathrm{KL}$ staging and further figuring out a new staging system which called West Lake (WL) staging orchestrated with JSW on X-rays and $\mathrm{Cl}, \mathrm{BML}$ on MRI.

\section{Methods}

\section{Patients enrollment}

A cross-section study had been undertaken. A total of 220 patients diagnosed with KOA in our institution from 2013 to 2018 were retrieved on the hospital information system (HIS). Generally, the mean age was $53.68 \pm 13.39$, the majority was female. Respectively $25,62,109$ and 24 knees were assessed as KL I, II, III and IV.

The inclusive criteria were: 1 , knee OA; 2 , with both eligible MRI (magnetic field intensity> 1.5 Tesla) and $X$-rays (projected in weight-bearing standing position with knees in $20^{\circ}$ of flexion and the feet in $5^{\circ}$ of extorsion [9]) document on knee; 3 , with entire case record.

The exclusive criteria were: 1 , with at least one musculoskeletal comorbidity, including rheumatoid arthritis, hemophilia arthritis, ankylosing spondylitis, gout and other painful diseases of the knee joint; 2, with complications affecting knees, including psoriasis, syphilitic neuropathy, ochronotic arthritis, metabolic osteopathy, acute trauma, etc.; 3 , the radiographs were unclear.

\section{KL staging}

The KL staging system was described as follow: Grade 0, no changes. Grade 1, doubtful joint space narrow (JSN) and a little osteophyte. Grade 2, possible JSN and distinct osteophyte. Grade 3, multiple osteophytes and definite JSN, some sclerosis and possible deformity of bone ends. Grade 4, large osteophytes, marked JSN, severe sclerosis, and deformity of bone ends [10].

Although there were some deficiencies on $\mathrm{KL}$ staging, it was still the recognized staging system on KOA. In order to reduce the error of KL staging caused by its shortcomings, two qualified orthopedic surgeons were invited to assess and record the KL stages of every patients, meanwhile the conflicting part were discussed by two surgeons to determine the final decision. 


\section{X-rays}

The narrowest JSW was measured on X-rays.

\section{MRI}

According to the Recht scores, $\mathrm{Cl}$ were divided into 4 grades: Grade 0, normal articular cartilage; Grade I, articular cartilage degeneration while no cartilage defect; Grade II, cartilage defect and the depth of cartilage defect was less than $50 \%$ of the full thickness; Grade III, the depth of cartilage defect was more than $50 \%$ while less than $100 \%$ of the full thickness; Grade IV, full-thickness cartilage defect and subchondral bone exposed [11].

It was difficult to measure the area of BML on MRI and the current WORMs scores were too complex, so referring to WORMs scores, the MRI of knee joint was divided into 15 regions, including lateral patellar section, medial patellar section, lateral-anterior femoral section, lateral-central femoral section, lateralposterior femoral section, medial-anterior femoral section, medial-central femoral section, medialposterior femoral section, lateral-anterior tibial section, lateral-central tibial section, lateral-posterior tibial section, medial-anterior tibial section, medial-central tibial section, medial-posterior tibial section and " $\mathrm{S}$ " section (The non-articulating portion of the tibial plateau beneath the tibial spines) [12]. Meanwhile the number of regions involved in BML were evaluated.

\section{Statistical Analysis}

Stata/MP, version 16.0 was used for statistical analysis and ROC curves were rendered by Medcalc, version 15.2.2. Statistical indicators included JSW, the grade of $\mathrm{Cl}$, the number of regions involved in $\mathrm{BML}$ as well as $\mathrm{KL}$ stages and $\mathrm{WL}$ stages assessed by two qualified orthopedic surgeons. All data were presented as mean $\pm S D$ except for $K L$ stages and WL stages which presented as ratio. The difference of those radiological parameters across the groups was calculated by T-test. Agreement Index (KAPPA) was used to analyze the consistency of KL stages and WL stages assessed by two qualified orthopedic surgeons. Receiver operating characteristic (ROC) curves were rendered to obtain the areas under the curves (AUC), Youden index and corresponding cut-off points. All reported P values are 2-tailed, with an alpha level of 0.05 indicating significance.

\section{Results}

\section{$\mathrm{KL} \mathrm{I} / \mathrm{II}$}

There were no statistical differences on JSW, $\mathrm{Cl}$ and BML between $\mathrm{KL} I$ and II. (Table 1)

\section{$\mathrm{KL} I \mathrm{I} / \mathrm{III}$}

Statistical differences were exerted between KL II and III on each item. (Table 1) 
The ROC curve of JSW showed the AUC was 0.99 , Youden index was 0.94 , cut-off point was $£ 5.1 \mathrm{~mm}$, meanwhile the corresponding sensitivity and specificity was $99.1 \%$ and $95.2 \%$. [Fig 1(a)]

The ROC curve of $\mathrm{Cl}$ indicated the AUC was 0.76 , Youden index was 0.38 , cut-off point was $>1$, simultaneously the corresponding sensitivity and specificity was $70.6 \%$ and $67.7 \%$. [Fig 1(b)]

The ROC curve of BML pointed the AUC was 0.71 , Youden index was 0.45 , cut-off point was $>2$, simultaneously the corresponding sensitivity and specificity was $59.6 \%$ and $85.5 \%$. [Fig 1 (c)]

\section{KL III/IV}

There were statistical differences on JSW, Cl and BML between KL III and IV. (Table 1)

The ROC curve of JSW showed the AUC was 0.96 , Youden index was 0.84 , cut-off point was $£ 3.2 \mathrm{~mm}$, meanwhile the corresponding sensitivity and specificity was $87.5 \%$ and $96.3 \%$. [Fig 2(a)]

The ROC curve of $\mathrm{Cl}$ indicated the AUC was 0.79 , Youden index was 0.58 , cut-off point was $>3$, simultaneously the corresponding sensitivity and specificity was $70.8 \%$ and $87.2 \%$. [Fig 2(b)]

The ROC curve of BML pointed the AUC was 0.74 , Youden index was 0.38 , cut-off point was $>4$, simultaneously the corresponding sensitivity and specificity was $70.8 \%$ and $67.0 \%$. [Fig 2(c)]

\section{Agreement Index (KAPPA)}

The Kappa coefficient of KL stages assessed by two qualified orthopedic surgeons was 0.31 while the Kappa coefficient of WL stages was 0.76. (Table 2)

Table 1 The results of T-test among KL I, II, III and IV

\begin{tabular}{|llll|}
\hline KL staging & JSW $($ Mean $\pm S D)(m m)$ & Cl $($ Mean $\pm S D)$ & BML $($ Mean $\pm S D)$ \\
\hline I $(n=25)$ & $5.89 \pm 0.99$ & $0.68 \pm 1.03$ & $1.76 \pm 1.30$ \\
\hline II $(n=62)$ & $6.13 \pm 0.80$ & $0.89 \pm 0.99$ & $1.65 \pm 1.19$ \\
\hline III $(n=109)$ & $4.17 \pm 0.58$ & $1.99 \pm 1.12$ & $3.69 \pm 2.87$ \\
\hline IV $(\mathrm{n}=24)$ & $1.82 \pm 1.32$ & $3.33 \pm 1.17$ & $6.50 \pm 3.23$ \\
\hline $\mathrm{KL} \mathrm{I/II} \mathrm{t,} \mathrm{P}$ & $-1.22,>0.05$ & $-0.87,>0.05$ & $0.40,>0.05$ \\
\hline $\mathrm{KL} \mathrm{II/III} \mathrm{t,} \mathrm{P}$ & $18.44,<0.05$ & $-6.46,<0.05$ & $-6.51,<0.05$ \\
\hline $\mathrm{KL} \mathrm{III/IV} \mathrm{t,} \mathrm{P}$ & $8.52,<0.05$ & $-5.28,<0.05$ & $-4.22,<0.05$ \\
\hline
\end{tabular}

Table 2 The results of Agreement Index (KAPPA) in KL and WL staging 


\begin{tabular}{|llllll|}
\hline & Expected Agreement & Agreement & Kappa & Z & P \\
\hline KL staging & $58.16 \%$ & $39.72 \%$ & 0.31 & 8.95 & $<0.05$ \\
\hline WL staging & $85.00 \%$ & $36.44 \%$ & 0.76 & 236.81 & $<0.05$ \\
\hline
\end{tabular}

\section{Discussion}

To our knowledge, this was the first study combining MRI and X-ray features to classify KOA grades. The reason of largely maintaining the structure of $\mathrm{KL}$ staging system instead of entirely subverting it was that X-ray was always the most available and convenient tool in the world for screening osteoarthritis [13]. In this study, the description of osteophytes in $\mathrm{KL}$ staging was removed except for grade 0 and I. For there was still a lack of an effective quantitative method to evaluate the number of osteophytes [14]. But surgeons just need to distinguish $\mathrm{KL} 0$ and I by determining whether there was osteophyte formation, so $\mathrm{KL} 0$ was easier to be distinguished from and not included in the study [7]. On the contrary, JSW was easier to be measured and confirmed that had a significant association with the severity of KOA as well as knee pain $[15,16]$. The mechanism of KOA could be summarized as follow: Abnormal mechanical loads were applied to the cartilage and transmitted to the subchondral bone, which led to microfractures of the subchondral bone, then an inflammatory response occurred and immune cells were recruited, which could activate bone growth factor and promotes subchondral bone reconstruction which led to the formation of BML finally [17-19]. Current studies suggest that the pathogenesis of KOA is mainly the lesion of the cartilage layer, meanwhile BML could inform on the degenerative state within the osteochondral unit [20,21]. In addition, BML has been known as a phenomenon to assess the severity and prognosis of $\mathrm{KOA}[22,23]$. So, $\mathrm{Cl}$ and $\mathrm{BML}$ were included in the study.

The study showed the KAPPA coefficient of two qualified orthopedic surgeons' KL stages was 0.31 which indicated their conclusions were less consistent. Previous literature uncovered intra-rater reliability ranging from 0.67 to 0.73 and hardship in distinguishing $\mathrm{KL}$ II and III by means of $\mathrm{KL}$ staging $[10,24]$. Guermazi proposed that $\mathrm{KL}$ staging had little information about the rate of cartilage degeneration through the comparison between same KOA patient's X-ray and MRI [25]. So, it was necessary to propose a new staging system so that every surgeon could agree on the severity of KOA.

In further research, we found there were no statistical differences on JSW, $\mathrm{Cl}$ and $\mathrm{BML}$ between $\mathrm{KL}$ I and II which let us to make the decision of merging them together. In fact, it was usually scrutinizing osteophytes by erudite orthopedic surgeons that work it out under this setting, recognizing $\mathrm{KL} \mathrm{I/II} \mathrm{by} \mathrm{X-ray}$ may be reluctant for universal orthopedic surgeons, which was supported by Adam [10]. The possible reason for the failed correlation between $\mathrm{Cl}$ and $\mathrm{KL} \mathrm{I/II} \mathrm{was} \mathrm{that} \mathrm{although} \mathrm{cartilage} \mathrm{was} \mathrm{merely} \mathrm{denatured}$ or with trivial defects, it has not been shown in the narrowing of joint space [26]. It is well known that the formation of BML was closely related to cartilage injuries, with mild cartilage damage, the vicious cycle 
of BML formation was less severe which caused the mild BML in $\mathrm{KL} \mathrm{I/II} \mathrm{[27].} \mathrm{Madry} \mathrm{et} \mathrm{al} \mathrm{[28]} \mathrm{have} \mathrm{also}$ put forward that merged $\mathrm{KL} \mathrm{I/II} \mathrm{as} \mathrm{early} \mathrm{KOA} \mathrm{for} \mathrm{their} \mathrm{trivial} \mathrm{difference} \mathrm{on} \mathrm{MRI} \mathrm{and} \mathrm{X-ray.}$

Dramatically, there were significant differences on JSW, Cl and BML between $\mathrm{KL}$ II and III as well as III and IV. ROC curves of JSW, Cl and BML showed the AUC was $0.99,0.76,0.71$ in $\mathrm{KL} \mathrm{II/III} \mathrm{and} 0.96,0.79,0.74$ in $\mathrm{KL}$ III/IV respectively which suggested high diagnostic value and JSW was the best indicator among them. When picked the maximum Youden index which meant optimal sensitivity and specificity, JSW $£ 5.1 \mathrm{~mm}, \mathrm{Cl}>1$ and $\mathrm{BML}>2$ were the cut-off points to differentiate $\mathrm{KL}$ II and III, and JSW $£ 3.2 \mathrm{~mm}, \mathrm{Cl}>3$ and $B M L>4$ were the cut-off points to distinguish KL IV from III. Although there was error in joint clearance measurements and people in different regions maid have different JSW, our study confirmed that compared with KL I and II, the JSW of KL III and IV were narrow and JSW of KL IV were the most serious one, which has also been verified by previous studies [29,30]. At present, no studies have explored the difference of $\mathrm{Cl}$ between $\mathrm{KL}$ II and III, while it was known that narrowing of the joint space could reflect the degree of cartilage wear, so cartilage damage was gradually aggravated with the increase of $\mathrm{KL}$ staging and Full-thickness wear of the cartilage was often present in KL IV for the reason of extreme narrowing or loss of JSW [31]. Since we used the number of regions involved in BML as an indicator, no data were found in the current literature to support our results. However, we have known that the area of BML were associated with the progression of KOA, meanwhile BML size was associated with longitudinal cartilage loss [32, 33].

According to the above research results, our group proposed WL staging system and the Kappa coefficient of WL stages assessed by two qualified orthopedic surgeons was 0.76 , which showed high consistency. It also indicated that surgeons had more consistent results with WL staging than with $\mathrm{KL}$ staging.

Our study still had several limitations. Firstly, we did not take into consideration of synovium, joint effusion, ligaments, meniscus and other imaging on MRI since they had no quantitative or semiquantitative evaluative methods. Secondly, given the inadequate following period, we could not verify the novel classification in terms of the related clinical symptoms, however we will publish our short-middle term results of this grading later.

\section{Conclusion}

In summary, The WL staging was described as follows: Grade 0, normal X-ray; Grade 1: X-ray shows osteophyte but no joint space narrow, normal MRI or MRI shows cartilage degeneration and only 1 or 2 sections are involved in BML; Grade 2: X-ray shows joint space narrow, MRI shows cartilage defect but no full-thickness cartilage defect, meanwhile 3 or 4 sections are involved in BML; Grade 3: X-ray shows serious joint space narrow even JSW disappeared, MRI shows full-thickness cartilage defect, more than 4 sections are involved in BML.

\section{Abbreviations}


KL: Kellgren - Lawrence; KOA: Knee osteoarthritis; WL: West Lake; JSW: Joint space widths; Cl: Cartilage injuries; BML: Bone marrow lesions; ROC: Receiver operating characteristic; AUC: the areas under the curves.

\section{Declarations}

\section{Ethics approval and consent to participate}

The study was approved by the First Affiliated Hospital of Zhejiang Chinese Medical University (2018-ZX026-01).

\section{Consent for publication}

All authors were consent for publication.

\section{Availability of data and materials}

The data of this study was available from the corresponding author on reasonable request.

\section{Competing interests}

All authors confirmed there were no competing interests.

\section{Funding}

The study was in part supported by the National Natural Science Foundation of China (grant nos. 81873324) and the project of Administration of Traditional Chinese Medicine of Zhejiang Province of China (grant no. 2018ZZ011).

\section{Authors' contributions}

Jiaxin Huang conceived the project and contributed to the study design and collected data. Jiaxin Huang and Xi Chen contributed to the statistical analysis. All authors contributed to drafted paper. Peijian Tong and Shuaijie Lv oversaw the project and approved the final version to be submitted.

\section{Acknowledgements}

The authors would like to thank staff and participants of the First Affiliated Hospital of Zhejiang Chinese Medical University.

\section{References}

1. Kolasinski SL, Neogi T, Hochberg MC, Oatis C, Guyatt G, Block J, et al. 2019 American College of Rheumatology/Arthritis Foundation Guideline for the Management of Osteoarthritis of the Hand, Hip, and Knee. Arthritis Care Res (Hoboken). 2020;72:149-62. 
2. Sharma L, Kwoh K, Lee JJ, Cauley J, Jackson R, Hochberg M, et al. Development and validation of risk stratification trees for incident slow gait speed in persons at high risk for knee osteoarthritis. Ann Rheum Dis. 2019;78:1412-9.

3. Tang X, Wang S, Zhan S, Niu J, Tao K, Zhang Y, et al. The Prevalence of Symptomatic Knee Osteoarthritis in China: Results From the China Health and Retirement Longitudinal Study. Arthritis Rheumatol. 2016;68:648-53.

4. Hunter DJ, Bierma-Zeinstra S. Osteoarthritis. Lancet. 2019;393:1745-59.

5. KELLGREN JH, LAWRENCE JS. Radiological assessment of osteo-arthrosis. Ann Rheum Dis. 1957;16:494-502.

6. Schiphof D, Boers M, Bierma-Zeinstra SM. Differences in descriptions of Kellgren and Lawrence grades of knee osteoarthritis. Ann Rheum Dis. 2008;67:1034-6.

7. Schiphof D, De Klerk B, Kerkhof H, Hofman A, Koes B, Boers M, et al. Impact of different descriptions of the Kellgren and Lawrence classification criteria on the diagnosis of knee osteoarthritis. Ann Rheum Dis. 2011;70:1422-7.

8. Oo WM, Linklater JM, Hunter DJ. Imaging in knee osteoarthritis. Curr Opin Rheumatol. 2017;29:86-95.

9. Luyten FP, Denti M, Filardo G, Kon E, Engebretsen L. Definition and classification of early osteoarthritis of the knee. Knee Surg Sports Traumatol Arthrosc. 2012;20:401-6.

10. Culvenor AG, Engen CN, Øiestad BE, Engebretsen L, Risberg MA. Defining the presence of radiographic knee osteoarthritis: a comparison between the Kellgren and Lawrence system and OARSI atlas criteria. Knee Surg Sports Traumatol Arthrosc. 2015;23:3532-9.

11. Recht MP, Kramer J, Marcelis S, Pathria MN, Trudell D, Haghighi P, et al. Abnormalities of articular cartilage in the knee: analysis of available MR techniques. Radiology. 1993;187:473-8.

12. Peterfy CG, Guermazi A, Zaim S, Tirman PF, Miaux Y, White D, et al. Whole-Organ Magnetic Resonance Imaging Score (WORMS) of the knee in osteoarthritis. Osteoarthritis Cartilage. 2004;12:177-90.

13. Duryea J, Neumann G, Niu J, Totterman S, Tamez J, Dabrowski C, et al. Comparison of radiographic joint space width with magnetic resonance imaging cartilage morphometry: analysis of longitudinal data from the Osteoarthritis Initiative. Arthritis Care Res (Hoboken). 2010;62:932-7.

14. Hakky M, Jarraya M, Ratzlaff C, Guermazi A, Duryea J. Validity and responsiveness of a new measure of knee osteophytes for osteoarthritis studies: data from the osteoarthritis initiative. Osteoarthritis Cartilage. 2015;23:2199-205.

15. Muraki S, Akune T, En-Yo Y, Yoshida M, Suzuki T, Yoshida $H$, et al. Joint space narrowing, body mass index, and knee pain: the ROAD study (OAC1839R1). Osteoarthritis Cartilage. 2015;23:874-81.

16. An BC, Fang K, Wang Y, Zeng YM, Dai KR. New variables for measuring joint space width to evaluate knee osteoarthritis. Chin Med J (Engl). 2011;124:3886-90.

17. Chen Y, Hu Y, Yu YE, Zhang X, Watts T, Zhou B, et al. Subchondral Trabecular Rod Loss and Plate Thickening in the Development of Osteoarthritis. J Bone Miner Res. 2018;33:316-27. 
18. Vaysbrot EE, Osani MC, Musetti MC, McAlindon TE, Bannuru RR. Are bisphosphonates efficacious in knee osteoarthritis? A meta-analysis of randomized controlled trials. Osteoarthritis Cartilage. 2018;26:154-64.

19. Weber A, Chan P, Wen C. Do immune cells lead the way in subchondral bone disturbance in osteoarthritis. Prog Biophys Mol Biol. 2019;148:21-31.

20. Showiheen S, Sun AR, Wu X, Crawford R, Xiao Y, Wellard RM, et al. Application of Metabolomics to Osteoarthritis: from Basic Science to the Clinical Approach. Curr Rheumatol Rep. 2019;21:26.

21. Muratovic D, Findlay DM, Cicuttini FM, Wluka AE, Lee YR, Edwards S, et al. Bone marrow lesions in knee osteoarthritis: regional differences in tibial subchondral bone microstructure and their association with cartilage degeneration. Osteoarthritis Cartilage. 2019;27:1653-62.

22. Felson DT, Lynch J, Guermazi A, Roemer FW, Niu J, McAlindon T, et al. Comparison of BLOKS and WORMS scoring systems part II. Longitudinal assessment of knee MRIs for osteoarthritis and suggested approach based on their performance: data from the Osteoarthritis Initiative. Osteoarthritis Cartilage. 2010;18:1402-7.

23. Lynch JA, Roemer FW, Nevitt MC, Felson DT, Niu J, Eaton CB, et al. Comparison of BLOKS and WORMS scoring systems part I. Cross sectional comparison of methods to assess cartilage morphology, meniscal damage and bone marrow lesions on knee MRI: data from the osteoarthritis initiative. Osteoarthritis Cartilage. 2010;18:1393-401.

24. Kohn MD, Sassoon AA, Fernando ND. Classifications in Brief: Kellgren-Lawrence Classification of Osteoarthritis. Clin Orthop Relat Res. 2016;474:1886-93.

25. Guermazi A, Roemer FW, Burstein D, Hayashi D. Why radiography should no longer be considered a surrogate outcome measure for longitudinal assessment of cartilage in knee osteoarthritis. Arthritis Res Ther. 2011;13:247.

26. Amin S, LaValley MP, Guermazi A, Grigoryan M, Hunter DJ, Clancy M, et al. The relationship between cartilage loss on magnetic resonance imaging and radiographic progression in men and women with knee osteoarthritis. Arthritis Rheum. 2005;52:3152-9.

27. Tanamas SK, Wluka AE, Pelletier JP, Martel-Pelletier J, Abram F, Wang Y, et al. The association between subchondral bone cysts and tibial cartilage volume and risk of joint replacement in people with knee osteoarthritis: a longitudinal study. Arthritis Res Ther. 2010;12:R58.

28. Madry H, Kon E, Condello V, Peretti GM, Steinwachs M, Seil R, et al. Early osteoarthritis of the knee. Knee Surg Sports Traumatol Arthrosc. 2016;24:1753-62.

29. Ratzlaff C, Ashbeck EL, Guermazi A, Roemer FW, Duryea J, Kwoh CK. A quantitative metric for knee osteoarthritis: reference values of joint space loss. Osteoarthritis Cartilage. 2018;26:1215-24.

30. Hellio Le Graverand MP, Mazzuca S, Duryea J, Brett A. Radiographic grading and measurement of joint space width in osteoarthritis. Rheum Dis Clin North Am. 2009;35:485-502.

31. Simoni P, Jamali S, Albert A, Totterman S, Schreyer E, Tamez-Peña JG, et al. Minimum joint space width (mJSW) of patellofemoral joint on standing "skyline" radiographs: test-retest reproducibility 
and comparison with quantitative magnetic resonance imaging (qMRI). Skeletal Radiol. 2013;42:1573-82.

32. Felson DT, McLaughlin S, Goggins J, LaValley MP, Gale ME, Totterman S, et al. Bone marrow edema and its relation to progression of knee osteoarthritis. Ann Intern Med. 2003;139:330-6.

33. Pang J, Driban JB, Destenaves G, Miller E, Lo GH, Ward RJ, et al. Quantification of bone marrow lesion volume and volume change using semi-automated segmentation: data from the osteoarthritis initiative. BMC Musculoskelet Disord. 2013;14:3.

\section{Figures}


Figure 1

The ROC curve of JSW (a), Cl (b), BML (c) between KL II and III
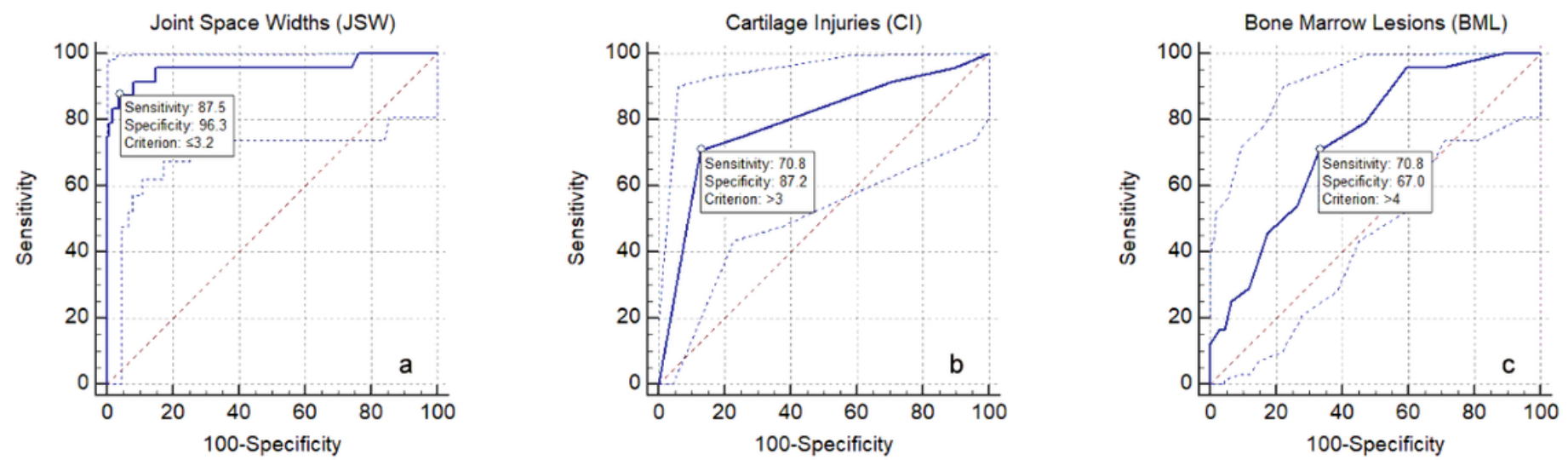

Figure 2

The ROC curve of JSW (a), Cl (b), BML (c) between KL III and IV

\section{Supplementary Files}


This is a list of supplementary files associated with this preprint. Click to download.

- DATA.xls 